\title{
Anterior cruciate ligament reconstruction using bone plug-free quadriceps tendon autograft: intermediate-term clinical outcome after 24-36 months
}

\author{
This article was published in the following Dove Press journal: \\ Open Access Journal of Sports Medicine \\ 20 November 2013 \\ Number of times this article has been viewed
}

\author{
Arndt P Schulz' \\ Vivien Lange ${ }^{2}$ \\ Justus Gille' \\ Christine Voigt ${ }^{3}$ \\ Susanne Fröhlich ${ }^{4}$ \\ Markus Stuhr' \\ Christian Jürgens ${ }^{5}$ \\ 'Department of Orthopedics, \\ Trauma, and Sports Medicine, \\ University Hospital Lübeck, \\ Lübeck, Germany; ${ }^{2}$ Department of \\ Rehabilitation, Sana Regio Klinikum, \\ Wedel, Germany; ${ }^{3}$ Department of \\ Sports Medicine and Arthroscopy, \\ Diakoniekrankenhaus Friederikenstift, \\ Hannover, Germany; ${ }^{4}$ Department of \\ Orthopedics, University of Rostock, \\ Rostock, Germany; ${ }^{5}$ Department of \\ Orthopedics, Trauma, and Sports \\ Medicine, BG Trauma Hospital \\ Hamburg, Hamburg, Germany
}

\begin{abstract}
Introduction: Although known as a possible graft option for decades, quadriceps tendon grafts have often been termed a second-line graft option. We report a consecutive case series using this method as the primary treatment line. The rationale for this study was to evaluate the midterm results of this method in a prospective and consecutive case series. The primary study question was to determine the clinical results 24-36 months after primary anterior cruciate ligament (ACL) reconstruction using a bone plug-free quadriceps tendon autograft fixed with bioabsorbable cross-pins.
\end{abstract}

Materials and methods: The study population included 55 patients, of whom 24 were female (43.6\%). The mean age at the index procedure was 31.7 years (15-58 years). All patients received an ACL construction using a bone block-free quadriceps tendon graft fixed with resorbable cross-pins. The postoperative regimen included partial weight-bearing for 3 weeks and flexion limited to $90^{\circ}$ for six weeks; an orthosis was not used. The mean follow-up duration was 29.5 months (24.3-38.5 months) after the index procedure. The International Knee Documentation Committee (IKDC) subjective score and examination form was assessed, as well as the Lysholm and Gillquist score and the Tegner activity index. The Rolimeter arthrometer was used to assess the anterior laxity of the knee.

Results: Graft harvesting was possible in all cases; a bony extension was never required. On average, graft length was measured at $8.8 \mathrm{~cm}(7.5-10 \mathrm{~cm})$. The mean IKDC subjective score at follow-up was 80.44 points (55.17-100 points, standard deviation [SD] 12.05). The mean preinjury Tegner activity index was $4.98(2-7)$ compared to a mean value of $4.16(2-7$, SD 0.8$)$ at follow-up. There was a mean loss of 0.82 index points. The average Lysholm and Gillquist score was 89 points $(65-100$, SD 17.7). Of the results, $89.1 \%$ were in the good or very good groups; in one case $(1.8 \%)$, the result was poor, while the rest were fair.

Conclusion: ACL reconstruction using a bone plug-free quadriceps tendon autograft achieved satisfactory results in a midterm review.

Keywords: ACL reconstruction, arthroscopic treatment, quadriceps tendon, clinical case series

\section{Introduction}

Although known as possible graft options for decades, ${ }^{1-5}$ quadriceps tendon grafts were often termed a second-line graft option until recently. ${ }^{6}$ Although the tensile strength is equal to patella grafts in biomechanical testing, it has been only in the past few years that the use of quadriceps tendon grafts has been more widespread. ${ }^{7}$ 
A Korean group first reported results of quadriceps grafts used with patellar bone plugs as an alternative graft in 2001, and as an alternative in a double-bundle technique in $2006 .{ }^{8,9}$ The technique was mentioned as a possible first-line treatment recently, and we reported results of our first series of bone plug-free patellar grafts in $2009 .{ }^{10}$ Since then, more authors have reported results using this technique as a firstline method, with or without bone plugs. ${ }^{11-16}$

The reason for our group to develop a treatment method using this implant was the disappointment with patellar tendon bone-tendon-bone grafts in many of our patients, especially those with kneeling pain. The fixation method used for the technique was bioabsorbable cross-pins from the beginning, a method that was developing at that time ${ }^{17-20}$ and which has been described for use with bone-free quadriceps grafts. $^{21}$

The rationale for this study was to evaluate the midterm results of this method in a prospective and consecutive case series. The hypothesis was that the technique achieves results comparable to the gold-standard techniques in literaturereview comparison. The primary study question was to determine the clinical results 24-36 months after primary anterior cruciate ligament (ACL) reconstruction using a bone plug-free quadriceps tendon autograft fixed with bioabsorbable cross-pins.

The results are reported in comparison to the uninjured contralateral side and to subjective items before the ACL injury. Furthermore, the results are compared to midterm results of the current gold-standard methods from the literature.

\section{Materials and methods Design}

Prospectively included in this study were consecutive patients referred to the orthopedic unit of a single center over a period of 24 months (2007-2008). All patients were operated on by or under the supervision of a single surgeon. It was therefore a single-arm, single-center cohort study.

Inclusion criteria were defined as:

- rupture of the ACL, requiring surgery due to instability

- patients of both sexes under the age of 60 years at the time of surgery.

Exclusion criteria were defined as:

- inability to give informed consent for the study

- local active or suspected infection, systemic diseases that might influence the study results

- ACL injury occurring during a work-related accident covered by the workers' injury-compensation scheme
- current or past injuries to the ipsi- or contralateral knee, possibly altering knee function, including previous ACL rupture

- patients with a concomitant posterior cruciate ligament injury (patients with a concomitant meniscal lesion, collateral ligament injury, or chondral lesions were not excluded).

The ethical committee of the University Lübeck approved the study protocol. In line with suggestions from the ethics committee, the study visit was insured against accidental injuries, and each patient received a sum of $€ 20$ for travel costs.

\section{Patients}

During the study period, 112 patients received the investigated treatment. Of these, 84 met the study-protocol inclusion and exclusion criteria and consented to take part in the study.

At the follow-up time point, twelve patients had moved and were not traceable, nine patients did not reply, even after a second contact letter, four patients withdrew their consent due to personal reasons, and three patients did not attend their study appointment.

So 56 patients were examined, but one patient then had to be excluded, as he had sustained a contralateral fracture of the tibial head. Therefore, 55 patients were included in the evaluation. The study population included 24 female patients $(43.6 \%)$. The mean age at the index procedure was 31.7 years (15-58 years). Forty-one patients $(74.5 \%)$ had sustained a sports injury, nine patients had had a fall not related to sports, five patients had suffered an accident at their home, and in three cases the ACL rupture had occurred during a road traffic accident.

The mean follow-up duration was 29.5 months (24.3-38.5 months) after the index procedure. In 28 cases, the right leg was injured. The mean body mass index (BMI) was $23.5 \mathrm{~kg} / \mathrm{m}^{2}\left(17.9-35.8 \mathrm{~kg} / \mathrm{m}^{2}\right)$.

\section{Concomitant injuries}

In 30 cases $(54.5 \%)$, an isolated rupture of the ACL was found, in nine cases a medial meniscal lesion was documented and treated (16.4\%), in five cases a lateral meniscal lesion $(9.1 \%)$ was documented and treated, and in three cases both (5.5\%). Two patients sustained an "unhappy triad" injury (3.6\%). In 16 patients, the meniscal lesion was treated with partial resection; twice the menisci were sutured. Cartilage lesions were documented in four cases (7.3\%). According to the Outerbridge classification, there were grade II lesions in two cases and grade III lesions in two cases. ${ }^{22}$ 


\section{Surgical technique}

The surgical technique was standardized: a tourniquet with a pressure of $350 \mathrm{mmHg}$ was used in all cases, as well as a single-shot antibiotic prophylaxis. About $3 \mathrm{~cm}$ proximal to the patella, a $4 \mathrm{~cm}$ incision was used for transplant harvesting of a part of the ipsilateral quadriceps tendon (see Figure 1A); the harvesting started then in flexion of the knee in the proximal part, so that in cases of a very short tendon, a bone block of the kneecap in the distal part could be used. The median part of the tendon was then harvested towards the patella; this was performed while bringing the knee more and more into extension. By this technique, the incision can be kept very small. The knee joint was not opened. The quadriceps tendon was approximated with a number 1 Vicryl suture. The ends of the harvested graft were armed with a strong thread (eg, DePuy Mitek Orthocord ${ }^{\circledR}$; Johnson \& Johnson,
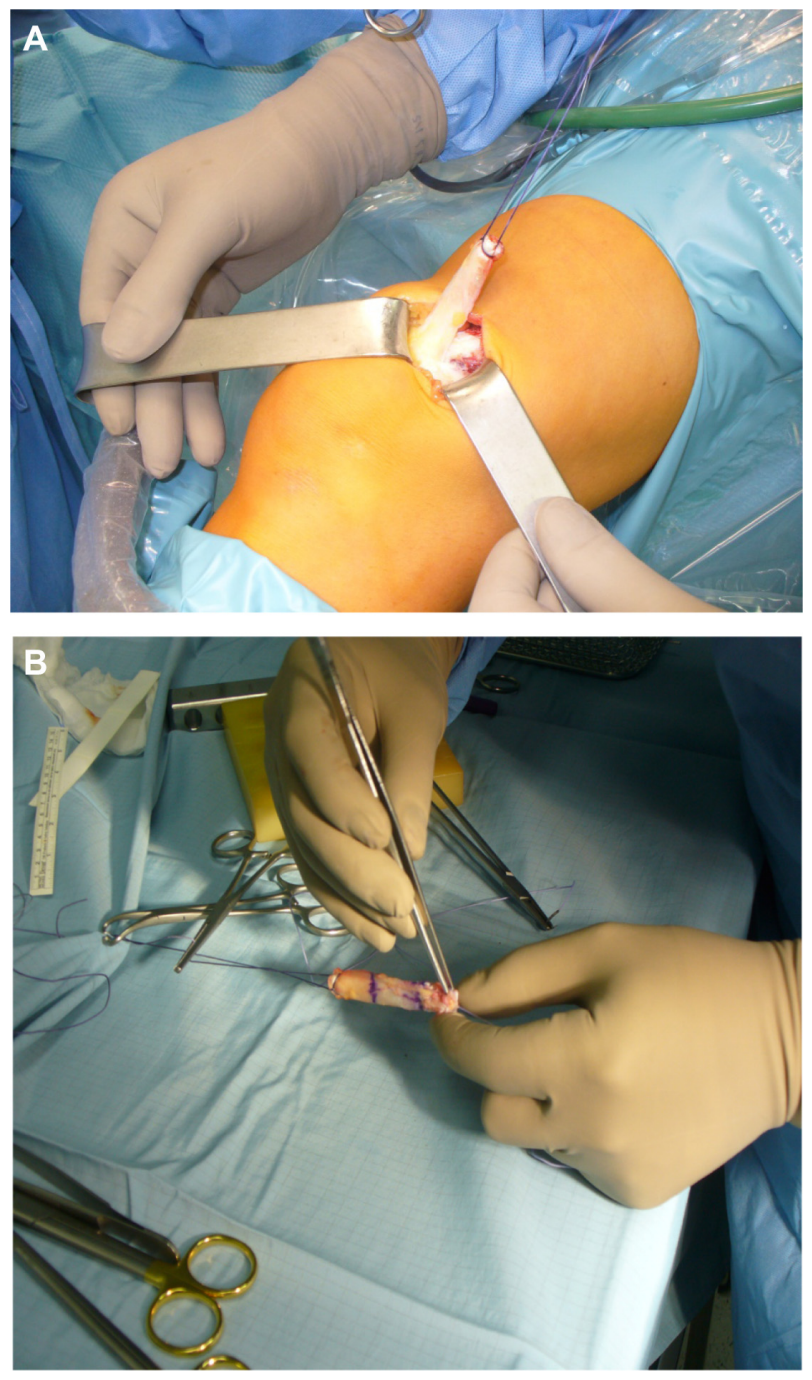

Figure I (A) Graft harvesting of the quadriceps tendon. (B) Interweaving of the graft ends to give a good support for the cross-pins.
New Brunswick, NJ, USA). After that, the proximal and distal ends of the graft were interweaved using a semiabsorbable suture on a side table (Orthocord) (Figure 1B).

Simultaneously, the procedure continued with resection of the ACL butts and drilling of the channels with a standard aiming device. The further procedure is similar to the technique utilizing hamstrings when the RigidFix ${ }^{\circledR}$ soft-tissue cross-pin system (Johnson \& Johnson) is used for anchoring; this has been described in detail in the past. ${ }^{23,24}$ In brief, the femoral and tibial cross-pins were placed with a femoral and tibial aiming device (Figure 2), then the graft was placed in the correct position. The femoral cross-pins were fixated first, followed by the tibial pins, which were fixated under graft tension. Graft implantation was performed transarthroscopically.

The postoperative regimen included partial weight bearing for 3 weeks and flexion limited to $90^{\circ}$ for 6 weeks; an orthosis was not used.

\section{Outcome parameters}

A clinical examination of the ipsi- and contralateral knee was performed, and all items of the International Knee Documentation Committee (IKDC) knee-examination form (excluding the radiographic evaluation) were used. In this, results are graded from $\mathrm{A}=$ normal to $\mathrm{D}=$ markedly abnormal. The lowest grade within a test item determines the group grade; the worst group grade determines the final evaluation.

The examiner was an independent physician not related to the surgical team (VL). The single-leg hop test used in this study has been in clinical use for more than 20 years; a positive correlation between muscular strength and jump distance has been described. ${ }^{25,26}$ The test method is in widespread use..$^{27-30}$ The IKDC subjective score was assessed,

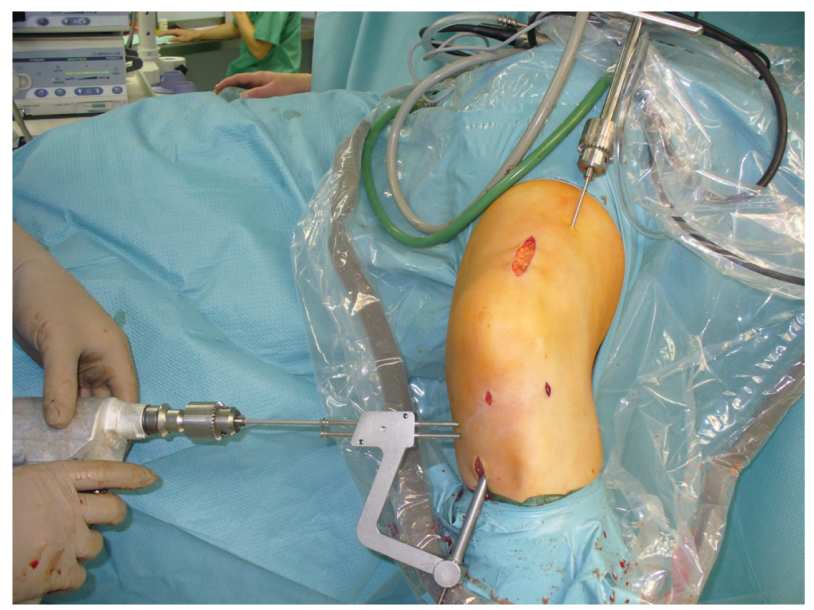

Figure 2 Placement of the tibial cross-pins before the graft is pulled in. 
as well as the Lysholm and Gillquist score and the Tegner activity index. ${ }^{27-29}$

The Rolimeter ${ }^{\circledR}$ arthrometer (Aircast; DJO Global, Vista, CA, USA) was used to assess the anterior laxity of the knee. This device has been described as having similar accuracy to the KT1000 Arthrometer $^{\circledR}$ (MedMetric, San Diego, CA, USA) with good intra- and intertester reliability. ${ }^{21,30,31} \mathrm{Graft}$ failure was defined as clinical failure confirmed by magnetic resonance imaging failure, or revision surgery with confirmed diagnosis.

\section{Statistical methods}

The descriptive statistics were obtained with SPSS (versions 16.0 and 20; IBM Corporation, Armonk, NY, USA). The Kolmogorov-Smirnov test was used to assess the normality of the distribution of the data. Depending on distribution and parameters, rank correlation coefficients using Pearson's or Spearman's tests or Kendall's tau were calculated. A samplesize calculation was not performed.

\section{Results \\ Procedure}

The mean skin-to-skin time was 89.2 minutes (65157 minutes). Graft harvesting was possible in all cases; a bony extension was never required. On average, graft length was measured as $8.8 \mathrm{~cm}(7.5-10 \mathrm{~cm})$. The diameter of the femoral graft site was on average $7.8 \mathrm{~mm}(6-9 \mathrm{~mm})$, of the tibia $9.2 \mathrm{~mm}(7-9.5 \mathrm{~mm})$. Cross-pin fixation was successful in all procedures.

\section{Complications}

There were no cases of deep vein thrombosis or infection. There was no case of quadriceps tendon rupture or graft rerupture. One patient $(1.8 \%)$ reported a numb area lateral to the graft-harvesting site. There were six cases of kneeling pain reported by patients $(11 \%)$. Three patients required a further procedure $(5.5 \%)$, twice for medial meniscal degeneration and once for a joint mobilization under anesthesia.

\section{Clinical examination}

Range of motion at follow-up examination compared to the contralateral knee revealed a mean restriction of $1.2^{\circ}\left(0^{\circ}-10^{\circ}\right)$ extension and $3.13^{\circ}\left(-10^{\circ}\right.$ to $\left.28^{\circ}\right)$ flexion. In 23 patients (41.8\%), the Lachman test was negative. In 21 cases (38.2\%), the test showed a $(+)$ result, in ten cases $(18.2 \%)$ a $(++)$ result. One case showed a $(+++)$ result; the pivot-shift test was negative in all cases.

\section{Single-leg hop test}

The single-leg hop test of the ipsilateral leg showed a mean of $84.08 \mathrm{~cm}(38.3-166.7 \mathrm{~cm})$. This equaled $89 \%$ of the mean distance of the contralateral side (49\%-140\%).

\section{Instrumental stability testing}

The mean anterior translation of the treated knee was $8.6 \mathrm{~mm}$, with the contralateral being $6.8 \mathrm{~mm}$ (Figure 3 ). The mean difference was therefore $1.8 \mathrm{~mm}$ ( -3 to $9.7 \mathrm{~mm}$, standard deviation [SD] 2.6).

\section{IKDC score}

The mean IKDC subjective score at follow-up was 80.44 points (55.17-100 points, SD 12.05).

\section{Tegner activity index}

The mean preinjury index was 4.98 (2-7) compared to a mean value of $4.16(2-7, \mathrm{SD} 0.8)$ at follow-up. There was a mean loss of 0.82 index points.

\section{Lysholm and Gillquist score}

The average Lysholm and Gillquist score was 89 points (65-100, SD 17.7). Of the results, 89.1\% were in the good or very good groups; in one case $(1.8 \%)$, the result was poor, while the rest were fair.

\section{IKDC knee-examination form}

According to the IKDC clinical examination grades, 13 knees (23.6\%) were graded as "normal (A)," 23 knees $(41.8 \%)$ as "nearly normal (B)," 17 knees (30.9\%) as "abnormal (C)," and two knees (3.6\%) as "severely abnormal (D)." The reason

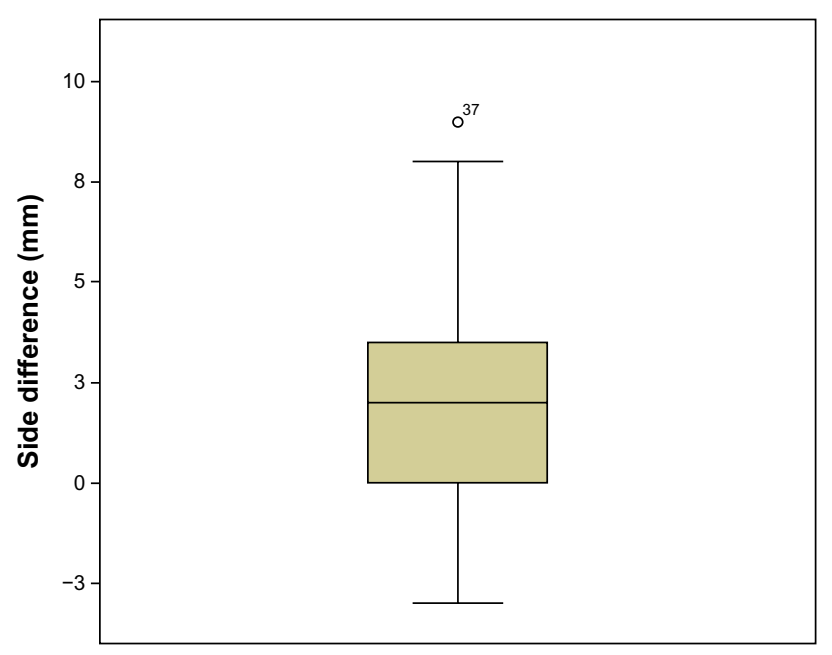

Figure 3 Box plot of the side difference in instrumental stability testing with the Rolimeter. 
for a $\mathrm{C}$ grade was a single-leg hop test of between $75 \%$ and $50 \%$ in the majority; the reason for a $\mathrm{D}$ grade was a flexion deficit of $28^{\circ}$ in one case and a third-degree positive anterior drawer test in the other. Statistical evaluation of the $A B$ versus the $C D$ groups found no statistical differences regarding age, sex, Tegner index, or Lysholm and Gillquist score. The only detectable difference in the groups was BMI ( $P=0.023$ ), with groups $\mathrm{C}$ and $\mathrm{D}$ having a mean $\mathrm{BMI}$ of 25.9 compared to 22.9 for the AB group.

\section{Discussion}

The principal finding of our study is that the technique offers a feasible graft, with an average graft length of about $8.8 \mathrm{~cm}$ and a low technical complication rate. The mean IKDC subjective score at follow-up was 80.44 points, and the mean Tegner activity index reduction was 0.82 index points. The average Lysholm and Gillquist score was 89 points, with $89.1 \%$ of the results in the good or very good groups.

In this study, we were able to show that this method is safe and reliable. Harvesting and transplantation was possible in all cases, including the cross-pin fixation. The graft length was sufficient in all cases; a bony extension into the patella, although technically possible, was never required. In our first case series, cross-pin fixation was not possible on two occasions, due to a small tibial head in female patients. ${ }^{32}$ Although a theoretical possibility, a rupture of the quadriceps tendon did not occur in our series.

The method of a bone plug-free tendon transplant was described for the first time in 2003; the technique of a bioabsorbable cross-pin fixation of the quadriceps free tendon was reported in $2005 .{ }^{33,34}$ We first reported results with this technique in 2009. ${ }^{32}$ Nevertheless, this method is far from reaching gold-standard status as the hamstring or patellar tendon-bone ( $\mathrm{tb})$ techniques have.

Regarding early postoperative complications in our study, we found no sign that the technique is inferior to other reported methods. ${ }^{24,29,35-45}$ Kneeling pain occurs more in a range that is comparable to the hamstring rather than the patellar tb technique. ${ }^{35,36}$

Clinical examination showed a satisfactory result, especially for extension movement, with a remaining slight extension deficit. The single-leg hop test showed very good results, indicating that a quadriceps muscle deficiency is not an automatic implication of this technique. ${ }^{37-41}$ Results of the test, with up to $140 \%$ hop distance of the nonoperated site, indicate a good, if not in some cases too focused, rehabilitation scheme.
In a randomized controlled trial, differences of anterior laxity to the contralateral site in instrumental testing have been reported, with a mean $2.7 \mathrm{~mm}$ (SD 2.1) for the hamstring and $2.7 \mathrm{~mm}$ (SD 2.2) for the patellar tb technique, ${ }^{35}$ with other authors reporting similar results. ${ }^{42,43}$

The mean subjective IKDC score determined in our study of 80.4 points is slightly lower than results achieved in a study that used quadriceps tendon with bone block, which reported a mean score of $86.1 \pm 15.8$ points. ${ }^{11}$ The Tegner activity index showed an activity level of 5 as the preinjury status; this is slightly lower than the value determined for a healthy population with a mean age of 41 years. ${ }^{44}$ Our findings at the follow-up examination, with an average activity index of 4.2, also appear to be lower than in many studies after hamstring or patellar tb procedures. ${ }^{7,45,46}$ On the other hand, our study population had a mean age of 32 years, and it has been shown that the Tegner activity level is inversely correlated with age. ${ }^{44}$

The average Lysholm and Gillquist score at follow-up was 89 points (65-100). In comparison, the aforementioned study examining values in a healthy population described a mean Lysholm and Gillquist score of 94. Of the results, $89.1 \%$ were in the good or very good groups in our study; this is a value that is comparable to results achieved in studies assessing hamstring or patellar tb techniques in a medium-term follow-up..$^{18,42,45-50}$ The aim of this study was to assess the medium-term results of a method - although in clinical use for a number of years now - that has never been reported in detail. ${ }^{9} 12,34,51$ The use of a bone-free quadriceps tendon transplant has a number of theoretical advantages when compared to hamstring or patellar tb transplants. Force reduction in flexion and internal rotation due to the loss of the harvested hamstrings has been described, ${ }^{5}$ and the formation of hematomas after graft harvesting has also been a criticism of this technique. ${ }^{52}$ The main problem of the patellar tb technique is anterior knee pain or kneeling pain. ${ }^{53-55}$ Judging by our study results, there is no evidence that these complications occur more frequently when using a bone plug-free quadriceps tendon graft.

\section{Limitations}

There was no control group in this study, and therefore we cannot conclude that this technique is superior to any other. There was a certain loss to follow-up that restricted data quality.

\section{Conclusion}

Comparing the study results of bone-free quadriceps ACL reconstruction with bioresorbable pin fixation with results 
of the uninjured side and reported results of the literature assessing gold-standard methods, we can conclude that the method is safe and reliable. Graft harvesting and fixation was possible in all cases. Results achieved were satisfactory at medium-term follow-up. Controlled trials comparing the technique directly with others would be expedient.

\section{Disclosure}

There are no conflicts of interest to declare, and this study was not funded by any external source.

\section{References}

1. Blauth W. 2-Strip substitution-plasty of the anterior cruciate ligament with the quadriceps tendon. Unfallheilkunde. 1984;87(2):45-51. German.

2. Howe JG, Johnson RJ, Kaplan MJ, Fleming B, Jarvinen M. Anterior cruciate ligament reconstruction using quadriceps patellar tendon graft. Part I. Long-term followup. Am J Sports Med. 1991;19(5):447-457.

3. Kaplan MJ, Howe JG, Fleming B, Johnson RJ, Jarvinen M. Anterior cruciate ligament reconstruction using quadriceps patellar tendon graft. Part II. A specific sport review. Am J Sports Med. 1991;19(5): $458-462$.

4. Kornblatt I, Warren RF, Wickiewicz TL. Long-term followup of anterior cruciate ligament reconstruction using the quadriceps tendon substitution for chronic anterior cruciate ligament insufficiency. Am J Sports Med. 1988;16(5):444-448.

5. Yasuda K, Tsujino J, Ohkoshi Y, Tanabe Y, Kaneda K. Graft site morbidity with autogenous semitendinosus and gracilis tendons. Am J Sports Med. 1995;23(6):706-714.

6. Becker R, Röpke M, Nebelung W. Anterior cruciate ligament reconstruction - graft options and fixation techniques. Zentralbl Chir. 2002;127(10):842-849.

7. Kartus J, Magnusson L, Stener S, Brandsson S, Eriksson BI, Karlsson J. Complications following arthroscopic anterior cruciate ligament reconstruction. A 2-5-year follow-up of 604 patients with special emphasis on anterior knee pain. Knee Surg Sports Traumatol Arthrosc. 1999;7(1):2-8.

8. Kim DW, Kim JO, You JD, Kim SJ, Kim HK. Arthroscopic anterior cruciate ligament reconstruction with quadriceps tendon composite autograft. Arthroscopy. 2001;17(5):546-550.

9. Kim SJ, Jung KA, Song DH. Arthroscopic double-bundle anterior cruciate ligament reconstruction using autogenous quadriceps tendon. Arthroscopy. 2006;22(7):797. e791-e795.

10. Bennett C, Loper I. Graft choices and fixation types in anterior cruciate ligament reconstruction. Curr Orthop Pract. 2008;19(3):288-292.

11. Akoto R, Hoeher J. Anterior cruciate ligament (ACL) reconstruction with quadriceps tendon autograft and press-fit fixation using an anteromedial portal technique. BMC Musculoskelet Disord. 2012;13:161.

12. Eberhardt C, Jäger A, Schwetlick G, Rauschmann MA. History of surgery of the anterior cruciate ligament. Orthopade. 2002;31(8): 702-709. German.

13. Geib TM, Shelton WR, Phelps RA, Clark L. Anterior cruciate ligament reconstruction using quadriceps tendon autograft: intermediate-term outcome. Arthroscopy. 2009;25(12):1408-1414.

14. Kim SJ, Jo SB, Kim TW, Chang JH, Choi HS, Oh KS. A modified arthroscopic anterior cruciate ligament double-bundle reconstruction technique with autogenous quadriceps tendon graft: remnant-preserving technique. Arch Orthop Trauma Surg. 2009;129(3):403-407.

15. Kim SJ, Kumar P, Oh KS. Anterior cruciate ligament reconstruction: autogenous quadriceps tendon-bone compared with bone-patellar tendon-bone grafts at 2-year follow-up. Arthroscopy. 2009;25(2): $137-144$.
16. Mauch C, Arnold MP, Wirries A, Mayer RR, Friederich NF, Hirschmann MT. Anterior cruciate ligament reconstruction using quadriceps tendon autograft for adolescents with open physes - a technical note. Sports Med Arthrosc Rehabil Ther Technol. 2011;3(1):7.

17. Chandratreya AP, Aldridge MJ. Top tips for RIGIDfix femoral fixation. Arthroscopy. 2004;20(6): e59-e61.

18. Harilainen A, Sandelin J, Jansson KA. Cross-pin femoral fixation versus metal interference screw fixation in anterior cruciate ligament reconstruction with hamstring tendons: results of a controlled prospective randomized study with 2-year follow-up. Arthroscopy. 2005;21(1):25-33.

19. Zantop T, Weimann A, Rümmler M, Hassenpflug J, Petersen W. Initial fixation strength of two bioabsorbable pins for the fixation of hamstring grafts compared to interference screw fixation: single cycle and cyclic loading. Am J Sports Med. 2004;32(3):641-649.

20. Zantop T, Welbers B, Weimann A, et al. Biomechanical evaluation of a new cross-pin technique for the fixation of different sized bone-patellar tendon-bone grafts. Knee Surg Sports Traumatol Arthrosc. 2004;12(6): 520-527.

21. Papandreou MG, Antonogiannakis E, Karabalis C, Karliaftis K. Interrater reliability of Rolimeter measurements between anterior cruciate ligament injured and normal contra lateral knees. Knee Surg Sports Traumatol Arthrosc. 2005;13(7):592-597.

22. Rupp S, Seil R, Jäger A, Kohn D. Replacement of the anterior cruciate ligament with a patellar tendon transplant. Orthopade. 2002;31(8): 751-757. German.

23. Fu FH, Bennett CH, Lattermann C, Ma CB. Current trends in anterior cruciate ligament reconstruction. Part 1: Biology and biomechanics of reconstruction. Am J Sports Med. 1999;27(6):821-830.

24. Haas NP. Surgery of the cruciate ligament - an ever current topic. Chirurg. Sep 2000;71(9):1023. German.

25. Wolter D. Biocompatibility of carbon fibre and carbon fibre microparticles. Aktuelle Probl Chir Orthop. 1983;26:28-36.

26. Sekiya I, Muneta T, Ogiuchi T, Yagishita K, Yamamoto H. Significance of the single-legged hop test to the anterior cruciate ligamentreconstructed knee in relation to muscle strength and anterior laxity. Am J Sports Med. 1998;26(3):384-388.

27. Hefti F, Müller W. Current state of evaluation of knee ligament lesions. The new IKDC knee evaluation form. Orthopade. 1993;22(6): 351-362.

28. Lysholm J, Gillquist J. Evaluation of knee ligament surgery results with special emphasis on use of a scoring scale. Am J Sports Med. 1982;10(3):150-154.

29. Tegner Y, Lysholm J. Rating systems in the evaluation of knee ligament injuries. Clin Orthop Relat Res. 1985;(198):43-49.

30. Balasch H, Schiller M, Friebel H, Hoffmann F. Evaluation of anterior knee joint instability with the Rolimeter. A test in comparison with manual assessment and measuring with the KT-1000 arthrometer. Knee Surg Sports Traumatol Arthrosc. 1999;7(4):204-208.

31. Pollet V, Barrat D, Meirhaeghe E, Vaes P, Handelberg F. The role of the Rolimeter in quantifying knee instability compared to the functional outcome of ACL-reconstructed versus conservatively-treated knees. Knee Surg Sports Traumatol Arthrosc. 2005;13(1):12-18.

32. Laxdal G, Kartus J, Ejerhed L, et al. Outcome and risk factors after anterior cruciate ligament reconstruction: a follow-up study of 948 patients. Arthroscopy. 2005;21(8):958-964.

33. Theut PC, Fulkerson JP, Armour EF, Joseph M. Anterior cruciate ligament reconstruction utilizing central quadriceps free tendon. Orthop Clin North Am. 2003;34(1):31-39.

34. Antonogiannakis E, Yiannakopoulos CK, Hiotis I, Karabalis C, Babalis G. Arthroscopic anterior cruciate ligament reconstruction using quadriceps tendon autograft and bioabsorbable cross-pin fixation. Arthroscopy. 2005;21(7):894.

35. Kobayashi A, Higuchi H, Terauchi M, Kobayashi F, Kimura M, Takagishi K. Muscle performance after anterior cruciate ligament reconstruction. Int Orthop. 2004;28(1):48-51. 
36. Kramer J, Nusca D, Fowler P, Webster-Bogaert S. Knee flexor and extensor strength during concentric and eccentric muscle actions after anterior cruciate ligament reconstruction using the semitendinosus tendon and ligament augmentation device. Am J Sports Med. 1993;21(2):285-291.

37. Ageberg E, Zätterström R, Fridén T, Moritz U. Individual factors affecting stabilometry and one-leg hop test in 75 healthy subjects, aged 15-44 years. Scand J Med Sci Sports. 2001;11(1):47-53.

38. Eriksson K, Anderberg P, Hamberg P, et al. A comparison of quadruple semitendinosus and patellar tendon grafts in reconstruction of the anterior cruciate ligament. J Bone Joint Surg Br. 2001;83(3):348-354.

39. Järvelä T, Kannus P, Latvala K, Järvinen M. Simple measurements in assessing muscle performance after an ACL reconstruction. Int J Sports Med. 2002;23(3):196-201.

40. Pokar S, Wissmeyer T, Krischak G, Kiefer H, Kinzl L, Hehl G. Arthroscopically-assisted reconstruction of the anterior cruciate ligament with autologous patellar tendon replacement-plasty. 5 years results. Unfallchirurg. 2001;104(4):317-324. German.

41. Rudolph KS, Axe MJ, Snyder-Mackler L. Dynamic stability after ACL injury: who can hop? Knee Surg Sports Traumatol Arthrosc. 2000;8(5):262-269.

42. Barrack RL, Bruckner JD, Kneisl J, Inman WS, Alexander AH. The outcome of nonoperatively treated complete tears of the anterior cruciate ligament in active young adults. Clin Orthop Relat Res. 1990;(259):192-199.

43. Li RC, Wu Y, Maffulli N, Chan KM, Chan JL. Eccentric and concentric isokinetic knee flexion and extension: a reliability study using the Cybex 6000 dynamometer. Br J Sports Med. 1996;30(2):156-160.

44. Engelhardt M, Freiwald J, Rittmeister M. Rehabilitation after anterior cruciate ligament reconstruction. Orthopade. 2002;31(8):791-798 German.

45. Buss DD, Min R, Skyhar M, Galinat B, Warren RF, Wickiewicz TL. Nonoperative treatment of acute anterior cruciate ligament injuries in a selected group of patients. Am J Sports Med. 1995;23(2):160-165.
46. Zysk SP, Refior HJ. Operative or conservative treatment of the acutely torn anterior cruciate ligament in middle-aged patients. A follow-up study of 133 patients between the ages of 40 and 59 years. Arch Orthop Trauma Surg. 2000;120(1-2):59-64.

47. Andersson C, Odensten M, Gillquist J. Knee function after surgical or nonsurgical treatment of acute rupture of the anterior cruciate ligament: a randomized study with a long-term follow-up period. Clin Orthop Relat Res. 1991;(264):255-263.

48. Brückner H. A new method for plastic surgery of cruciate ligaments. Chirurg. 1966;37(9):413-414. German.

49. Kvist J, Gillquist J. Anterior tibial translation during eccentric, isokinetic quadriceps work in healthy subjects. Scand JMed Sci Sports. 1999;9(4):189-194.

50. Rose T, Hepp P, Venus J, Stockmar C, Josten C, Lill H. Prospective randomized clinical comparison of femoral transfixation versus bioscrew fixation in hamstring tendon ACL reconstruction - a preliminary report. Knee Surg Sports Traumatol Arthrosc. 2006;14(8): 730-738.

51. Santori N, Adriani E, Pederzini L. ACL reconstruction using quadriceps tendon. Orthopedics. 2004;27(1):31-35.

52. Strobel MJ, Schulz MS. Anterior cruciate ligament reconstruction with the semitendinosus-gracilis tendon transplant. Orthopade. 2002;31(8): 758-769. German.

53. Aglietti P, Buzzi R, D’Andria S, Zaccherotti G. Patellofemoral problems after intraarticular anterior cruciate ligament reconstruction. Clin Orthop Relat Res. 1993;(288):195-204.

54. Kleipool AE, Zij1 JA, Willems WJ. Arthroscopic anterior cruciate ligament reconstruction with bone-patellar tendon-bone allograft or autograft. A prospective study with an average follow up of 4 years. Knee Surg Sports Traumatol Arthrosc. 1998;6(4):224-230.

55. Sachs RA, Daniel DM, Stone ML, Garfein RF. Patellofemoral problems after anterior cruciate ligament reconstruction. Am J Sports Med. 1989;17(6):760-765.
Open Access Journal of Sports Medicine

\section{Publish your work in this journal}

Open Access Journal of Sports Medicine is an international, peer-reviewed, open access journal publishing original research, reports, reviews and commentaries on all areas of sports medicine. The manuscript management system is completely online and includes a very quick and fair peer-review system.

\section{Dovepress}

Visit http://www.dovepress.com/testimonials.php to read real quotes from published authors. 